\title{
Complicações na gravidez e diabetes mellitus na gestação: dados de morbidade e mortalidade no Brasil
}

Pregnancy complications and diabetes mellitus during pregnancy: morbidity and mortality data in Brazil

Complicaciones del embarazo y diabetes mellitus durante el embarazo: datos de morbilidad y mortalidad en Brasil

Ekaterini Cruz Perivolaris ORCID: https://orcid.org/0000-0002-7946-2851 Universidade Paulista, Brasil E-mail: ekateriniperivolaris@gmail.com

Samara Kelly da Silva Cavalcante ORCID: https://orcid.org/0000-0002-9799-8959 Centro Universitário Católica de Quixadá, Brasil E-mail: samarakelly0804@hotmail.com

Maria Natália Cavalcante da Silva ORCID: https://orcid.org/0000-0002-1036-5148 Centro Universitário Católica de Quixadá, Brasil E-mail: ncavalcante454@gmail.com

João Pedro Silva Teixeira ORCID: https://orcid.org/0000-0003-4240-6212 Centro Universitário Católica de Quixadá, Brasil

E-mail: Pedro00tri@hotmail.com

Vitória Ferreira Silva

ORCID: https://orcid.org/0000-0003-2628-7188 Centro Universitário Católica de Quixadá, Brasil

E-mail: vitoriasilvaferreira@outlook.com

Érika Matias Pinto Dinelly

ORCID: https://orcid.org/0000-0003-2786-3132 Centro Universitário Católica de Quixadá, Brasil E-mail: erikamatias@unicatolicaquixada.edu.br

\begin{abstract}
Resumo
A assistência pré-natal é de grande importância para uma gestação saudável, prevenindo e detectando precocemente possíveis complicações inerentes à gravidez e diminuindo, assim, a morbimortalidade materna e fetal. Sendo uma das intercorrências mais prevalentes, a diabetes mellitus gestacional tem grande impacto na saúde materno-fetal e pode causar complicações a curto e longo prazo. Com o fim de verificar a incidência e a mortalidade das complicações da gravidez, do parto e do puerpério e a mortalidade decorrente de diabetes mellitus na gestação no Brasil. Realizou-se a coleta de dados no Departamento de Informática do Sistema Único de Saúde (DATASUS) do período de 2017 a 2019 , baseando-se no Sistema de Informações Hospitalares do SUS (SIH/SUS) e no Sistema de Informações sobre Mortalidade (SIM). Diante disso, foi obtido que na mortalidade materna decorrente da diabetes mellitus na gravidez no Brasil houve um aumento de cerca de 18,18\% de 2014 para 2015 e de $15,38 \%$ de 2015 a 2016. Já de 2016 a 2017 , houve um decréscimo percentual de $40 \%$, seguido de um aumento de $40 \%$ entre 2017 e 2018 e posterior decréscimo entre 2018 e 2019 de 26,66\%. Em suma, a diabetes mellitus gestacional não foi considerada a maior responsável pelos óbitos no período estudado, no entanto, é um distúrbio que deve ser tratado e detectado precocemente, devido ao fato de que pode levar ao desenvolvimento de complicações tanto à gestante, quanto ao feto.
\end{abstract}

Palavras-chave: Diabetes gestacional; Morbidade; Mortalidade.

\begin{abstract}
Prenatal care is of great importance for a healthy pregnancy, preventing and early detecting possible complications inherent to pregnancy and thus reducing maternal and fetal morbidity and mortality. As one of the most prevalent complications, gestational diabetes mellitus has a great impact on maternal-fetal health and can cause short- and longterm complications. In order to verify the incidence and mortality of complications of pregnancy, childbirth and the puerperium and mortality due to diabetes mellitus during pregnancy in Brazil. Data collection was carried out in the Information Technology Department of the Unified Health System (DATASUS) from 2017 to 2019, based on the SUS Hospital Information System (SIH/SUS) and the Mortality Information System (SIM ). Therefore, it was found
\end{abstract}


that maternal mortality due to diabetes mellitus in pregnancy in Brazil there was an increase of about $18.18 \%$ from 2014 to 2015 and $15.38 \%$ from 2015 to 2016. From 2016 to 2017, there was an increase percentage decrease of 40\%, followed by an increase of $40 \%$ between 2017 and 2018 and a subsequent decrease between 2018 and 2019 of $26.66 \%$. In short, gestational diabetes mellitus was not considered the main cause of deaths in the period studied, however, it is a disorder that must be treated and detected early, due to the fact that it can lead to the development of complications for both the pregnant woman and the fetus.

Keywords: Gestational diabetes; Morbidity; Mortality.

\section{Resumen}

La atención prenatal es de gran importancia para un embarazo saludable, previniendo y detectando precozmente las posibles complicaciones inherentes al embarazo y reduciendo así la morbimortalidad materna y fetal. Como una de las complicaciones más prevalentes, la diabetes mellitus gestacional tiene un gran impacto en la salud materno-fetal y puede ocasionar complicaciones a corto y largo plazo. Con el fin de verificar la incidencia y mortalidad de las complicaciones del embarazo, parto y puerperio y mortalidad por diabetes mellitus durante el embarazo en Brasil. La recolección de datos se realizó en el Departamento de Tecnología de la Información del Sistema Único de Salud (DATASUS) de 2017 a 2019, con base en el Sistema de Información Hospitalaria del SUS (SIH / SUS) y el Sistema de Información de Mortalidad (SIM). Por lo tanto, se encontró que la mortalidad materna por diabetes mellitus en el embarazo en Brasil hubo un aumento de aproximadamente 18,18\% de 2014 a 2015 y 15,38\% de 2015 a 2016. De 2016 a 2017, hubo un aumento porcentual de disminución de 40\%, seguido de un aumento del $40 \%$ entre 2017 y 2018 y una posterior disminución entre 2018 y 2019 del 26,66\%. En resumen, la diabetes mellitus gestacional no fue considerada la principal causa de muerte en el período estudiado, sin embargo, es un trastorno que debe ser tratado y detectado de manera precoz, debido a que puede conducir al desarrollo de complicaciones tanto para la gestante. mujer y el feto.

Palabras clave: Diabetes gestacional; Morbosidad; Mortalidad.

\section{Introdução}

A assistência pré-natal é de grande importância para uma gestação saudável, prevenindo e detectando precocemente possíveis complicações inerentes à gravidez e diminuindo, assim, a morbimortalidade materna e fetal (Tomasi et al., 2017).

Desse modo, a mortalidade materna se torna um indicador de saúde e pode representar as disparidades nos cuidados maternos em diferentes regiões do Brasil e do mundo (Laurenti et al., 2004; Grandi et al., 2019). Frente a isso, a melhora da saúde da gestante e a consequente redução de mortalidade materna estão entre as principais metas de conferências internacionais e tornam-se, no Brasil, o quinto Objetivo de Desenvolvimento do Milênio (ODM). Este tinha como propósito, até 2015, reduzir a mortalidade materna a três quartos do nível observado em 1990, entretanto, essa meta não foi alcançada. Então, o novo objetivo será reduzir a razão de mortalidade materna (RMM) para 20/100.000 nascidos vivos até 2030 (Brasil, 2012; Silva et al., 2018; Roma, 2019).

Os índices de mortalidade no Brasil são estimados pelo Sistema de Informações sobre Mortalidade, que é administrado pelo Ministério da Saúde (SIM/MS), cuja abrangência é de, aproximadamente, $85 \%$ do país. As regiões Norte e Nordeste são as que apresentam menor cobertura, já no Sul e no Sudeste a abrangência é quase completa (Laurenti et al., 2004; Brasil, 2008).

A presença de complicações que ocorrem no período da gravidez, parto ou puerpério está diretamente relacionada à falta de atenção a cuidados pré-natais, tendo em vista que, com o diagnóstico precoce de alterações metabólicas ou doenças relacionadas a fatores maternos, diminuem-se os riscos de complicações maternas e fetais (Leite et al., 2012).

A morbimortalidade materna é compreendida pelo capítulo XV da Classificação Internacional de Doenças (CID-10), que padroniza e codifica as doenças e os problemas relacionados à saúde (Laurenti et al., 2004). No caso das morbidades maternas mais frequentes, estão as infecções, doenças hipertensivas, diabetes e hemorragias, que podem levar ao óbito (Walker et al., 2019). Sendo uma das intercorrências mais prevalentes, a diabetes mellitus gestacional (DMG) tem grande impacto na saúde materno-fetal e pode causar complicações a curto e longo prazo (Schoenaker et al., 2016; Moura et al., 2018). 
Neste sentido, torna-se relevante pontuar a definição de diabetes mellitus gestacional, considerando sua frequência e importância no que tange à morbimortalidade materna. A DMG é caracterizada pela intolerância a carboidratos diagnosticada pela primeira vez no período da gravidez, podendo ou não persistir após o parto. Esse distúrbio metabólico tem prevalência global estimada entre 1 e 14\% e a média brasileira é de, aproximadamente, 7\% (Hunt, 2008; Packer, 2016; Battarbee et al., 2020).

Essa alteração metabólica pode causar diversas complicações tanto para as gestantes, quanto para os fetos. Nas gestantes, aumentam-se os riscos de desenvolverem pré-eclâmpsia, diabetes tipo II, doenças cardiovasculares e síndrome metabólica, e podem levar ao parto prematuro e cesárea. Já no caso dos fetos, elevam-se as chances de aparecimento de malformações congênitas nos sistemas esquelético, cardíaco e neurológico, além de poderem manifestar a macrossomia e hiperbilirrubinemia e, junto a isso, cresce a probabilidade de serem diabéticos tipo II na fase adulta (Galerneau \& Inzucchi, 2004; Reece, 2010; Mohsin et al., 2016).

Tendo em vista o que foi exposto, o objetivo deste trabalho foi verificar a incidência de morbidade e de mortalidade resultantes de complicações da gravidez no Brasil, ressaltando a mortalidade materna decorrente do diabetes mellitus na gestação.

\section{Metodologia}

Trata-se de um estudo de caráter descritivo (Pereira, Shitsuka, Parreira \& Shitsuka, 2018), realizado a partir de uma coleta de dados do Departamento de Informática do Sistema Único de Saúde (DATASUS), baseando-se no Sistema de Informações Hospitalares do SUS (SIH/SUS) e no Sistema de Informações sobre Mortalidade (SIM).

O estudo incluiu todas as mulheres que foram internadas em hospitais do SUS com complicações da gravidez e do parto, segundo o capítulo XV da Classificação Internacional de Doenças (CID-10), no período de janeiro de 2014 a dezembro de 2019, por região do Brasil.

Foi incluído também o número de óbitos maternos, de acordo com as categorias O20-O29, do capítulo XV da CID-10, entre os anos de 2014 a 2019. Os dados de mortalidade foram divididos tanto por região do Brasil, quanto por ano de óbito. Os resultados foram apresentados sobre forma quantitativa, por meio de cálculos estatísticos de porcentagem, a partir do levantamento de dados das plataformas utilizadas.

\section{Resultados}

Em relação à morbidade decorrente de complicações da gravidez e do parto no Brasil, segundo o Capítulo XV da CID-10, observou-se, inicialmente, uma diminuição, seguida de um pequeno aumento no número de casos. Comparando o ano de 2014 com 2015, houve um decréscimo de cerca de 0,64\%, com uma diminuição de 4.048 casos. Já de 2015 para 2016, houve um decréscimo de 5,22\%, com a redução de 32.898 casos. Entretanto, de 2016 para 2017, houve um aumento de 0,14\%, com o acréscimo de 864 ocorrências. Já entre os anos de 2017-2018 e 2018-2019, houve um aumento de 4,24\% e 0,22\%, respectivamente (Tabela 1). 
Tabela 1 - Morbidade Hospitalar do SUS - por local de residência - Brasil. Internações por Ano processamento e Região. Capítulo CID-10: XV. Gravidez parto e puerpério. Lista Morbidade CID-10: Outras complicações da gravidez e do parto. Período: 2014-2019.

\begin{tabular}{ccccccc}
\hline $\begin{array}{c}\text { Ano } \\
\text { processamento }\end{array}$ & $\begin{array}{c}\text { Região } \\
\text { Norte }\end{array}$ & $\begin{array}{c}\text { Região } \\
\text { Nordeste }\end{array}$ & $\begin{array}{c}\text { Região } \\
\text { Sudeste }\end{array}$ & $\begin{array}{c}\text { Região } \\
\text { Sul }\end{array}$ & $\begin{array}{c}\text { Região } \\
\text { Centro-Oeste }\end{array}$ & Total \\
\hline $\mathbf{2 0 1 4}$ & 71.713 & 158.998 & 241.553 & 101.822 & 60.161 & $\mathbf{6 3 4 . 2 4 7}$ \\
\hline $\mathbf{2 0 1 5}$ & 71.023 & 157.479 & 243.483 & 99.913 & 58.301 & $\mathbf{6 3 0 . 1 9 9}$ \\
\hline $\mathbf{2 0 1 6}$ & 65.643 & 147.940 & 235.095 & 92.354 & 56.269 & $\mathbf{5 9 7 . 3 0 1}$ \\
\hline $\mathbf{2 0 1 7}$ & 66.797 & 147.485 & 241.177 & 85.367 & 57.339 & $\mathbf{5 9 8 . 1 6 5}$ \\
\hline $\mathbf{2 0 1 8}$ & 67.828 & 163.496 & 246.199 & 85.050 & 60.993 & $\mathbf{6 2 3 . 5 6 6}$ \\
\hline $\mathbf{2 0 1 9}$ & 70.665 & 169.067 & 241.141 & 83.347 & 60.731 & $\mathbf{6 2 4 . 9 5 1}$ \\
\hline Total & $\mathbf{4 1 3 . 6 6 9}$ & $\mathbf{9 4 4 . 4 6 5}$ & $\mathbf{1 . 4 4 8 . 6 4 8}$ & $\mathbf{5 4 7 . 8 5 3}$ & $\mathbf{3 5 3 . 7 9 4}$ & $\mathbf{3 . 7 0 8 . 4 2 9}$ \\
\hline
\end{tabular}

Fonte: Ministério da Saúde - Sistema de Informações Hospitalares do SUS (SIH/SUS) (2021).

Já o número de ocorrências na região Norte sofreu uma diminuição de 0,96\% de 2014 para 2015 e de 7,58\% de 2015 para 2016. Contanto, de 2016 a 2017, sofreu um aumento de 1,76\%, e, comparando os anos de 2017 e 2019 , houve um aumento de 5,79\%. Já na região Nordeste, notou-se um decréscimo de 0,96\%, 6,06\% e de 0,31\% entre os anos de 2014 a 2017 , seguido de um aumento de $10,85 \%$ e 3,41\% entre 2017 a 2019.

O Sudeste apresenta o maior número de casos, totalizando 1.448 .648 de janeiro de 2014 a dezembro de 2019, havendo um acréscimo percentual de 0,80\% entre 2014-2015, um decréscimo de 3,45\% entre 2015-2016, novamente um aumento de 2,59\% entre 2016-2017 e de 2,08\% entre 2017-2018 e uma diminuição de 2,05\% entre 2018-2019. Por outro lado, no Sul do Brasil há, predominantemente, decréscimos percentuais: de 1,88\%, 7,57\%, 7,57\%, 0,37\% e 2,00\% de 2014 a 2019 (Tabela 1).

O Centro-Oeste é a região que possui os menores números de complicações gestacionais e do parto durante esse período, com a soma de 353.794 casos. Esses números sofreram uma diminuição de 3,09\% e de 3,49\%, entre 2014-2015 e 2015-2016, respectivamente, porém, aumentaram 1,90\% e 6,37\% de 2016 a 2017 e de 2017 a 2018 e sofreu uma pequena diminuição de $0,43 \%$ entre 2018 e 2019.

Na Tabela 2, após analisar os dados de mortalidade decorrente de transtornos maternos relacionados predominantemente à gravidez, observou-se que a maior causa de óbito é a infecção do trato geniturinário na gravidez (O23), responsável por $47 \%$ dos casos, seguida de assistência materna por outras complicações ligadas predominantemente à gravidez (O26), responsável por 22,77\% dos casos, que pode ser representativa de: herpes gestacional; síndrome da hipotensão materna; ganho excessivo de peso na gravidez; ganho de peso insuficiente na gravidez; assistência à gravidez por motivo de abortamento habitual; retenção de dispositivo intrauterino contraceptivo (DIU) na gravidez; transtornos do fígado na gravidez no parto e no puerpério; subluxação da sínfise (púbica) na gravidez, parto e puerpério; outras afecções especificadas, ligadas a gravidez; e afecções ligadas a gravidez, não especificadas. O terceiro maior causador de óbito materno, segundo o SIM, é a diabetes mellitus na gestação, equivalendo 15,32\% do total de óbitos no período de 2014 a 2019. 
Tabela 2 - Óbitos de mulheres em idade fértil e óbitos maternos - Brasil. Óbitos maternos por Categoria CID-10 e Ano do Óbito. Capítulo CID-10: XV. Gravidez parto e puerpério. Grupo CID-10: Outros transtornos maternos relacionados predominantemente à gravidez. Período: 2014-2019.

\begin{tabular}{|c|c|c|c|c|c|c|c|}
\hline Categoria CID-10 & 2014 & 2015 & 2016 & 2017 & 2018 & 2019 & Total \\
\hline O20 Hemorragia do início da gravidez & 3 & 1 & - & 2 & 2 & 1 & 9 \\
\hline O21 Vômitos excessivos na gravidez & 2 & 1 & 2 & - & 6 & 4 & 15 \\
\hline $\begin{array}{l}\text { O22 Complicações venosas na } \\
\text { gravidez }\end{array}$ & 3 & 7 & 6 & 10 & 4 & 6 & 36 \\
\hline $\begin{array}{c}\text { O23 Infecções do trato geniturinário } \\
\text { na gravidez }\end{array}$ & 46 & 36 & 39 & 34 & 39 & 33 & 227 \\
\hline O24 Diabetes mellitus na gravidez & 11 & 13 & 15 & 9 & 15 & 11 & 74 \\
\hline O25 Desnutrição na gravidez & 1 & 1 & 1 & 1 & - & - & 4 \\
\hline $\begin{array}{l}\text { O26 Assistência materna por outras } \\
\text { complicações ligadas a gravidez }\end{array}$ & 13 & 23 & 15 & 20 & 18 & 21 & 110 \\
\hline $\begin{array}{l}\text { O29 Complicações de anestesia } \\
\text { administrada durante gravidez }\end{array}$ & 2 & - & 1 & 1 & 1 & 3 & 8 \\
\hline Total & 81 & 82 & 79 & 77 & 85 & 79 & 483 \\
\hline
\end{tabular}

Fonte: MS/SVS/CGIAE - Sistema de Informações sobre Mortalidade - SIM (2021).

Em se tratando da mortalidade materna decorrente da diabetes mellitus na gravidez no Brasil, notou-se um aumento de cerca de 18,18\% de 2014 para 2015 e de 15,38\% de 2015 a 2016. Já de 2016 a 2017, houve um decréscimo percentual de $40 \%$, seguido de um aumento de 40\% entre 2017 e 2018 e posterior decréscimo entre 2018 e 2019 de 26,66\% (Tabela 3).

Já quanto à distribuição regional, observando na Figura 1, o Sudeste apresenta maiores números de mortalidade, correspondentes a 34 casos, seguido do Nordeste, com 22 casos.

Tabela 3 - Óbito de mulheres em idade fértil e óbitos maternos - Brasil. Óbitos maternos por ano do óbito e região. Capítulo CID-10: XV Gravidez, parto e puerpério. Capítulo CID-10: O24 Diabetes mellitus na gravidez. Período: 2014-2019.

\begin{tabular}{ccccccc}
\hline $\begin{array}{c}\text { Ano do } \\
\text { Óbito }\end{array}$ & $\begin{array}{c}\text { Região } \\
\text { Norte }\end{array}$ & $\begin{array}{c}\text { Região } \\
\text { Nordeste }\end{array}$ & $\begin{array}{c}\text { Região } \\
\text { Sudeste }\end{array}$ & $\begin{array}{c}\text { Região } \\
\text { Sul }\end{array}$ & $\begin{array}{c}\text { Região } \\
\text { Centro-Oeste }\end{array}$ & Total \\
\hline $\mathbf{2 0 1 4}$ & 2 & 2 & 5 & 1 & 1 & $\mathbf{1 1}$ \\
\hline $\mathbf{2 0 1 5}$ & - & 4 & 5 & 2 & 2 & $\mathbf{1 3}$ \\
\hline $\mathbf{2 0 1 6}$ & 2 & 4 & 7 & 2 & 1 & $\mathbf{1 5}$ \\
\hline $\mathbf{2 0 1 7}$ & - & 3 & 4 & 1 & - & $\mathbf{9}$ \\
\hline $\mathbf{2 0 1 8}$ & 2 & 5 & 8 & - & - & $\mathbf{1 1}$ \\
\hline $\mathbf{2 0 1 9}$ & 1 & 4 & 5 & 1 & $\mathbf{4}$ & $\mathbf{7 4}$ \\
\hline Total & $\mathbf{7}$ & $\mathbf{2 2}$ & $\mathbf{3 4}$ & $\mathbf{7}$ & &
\end{tabular}

Fonte: MS/SVS/CGIAE - Sistema de Informações sobre Mortalidade - SIM (2021). 
Figura 1 - Óbito de mulheres em idade fértil e óbitos maternos no Brasil, decorrentes de diabetes mellitus na gravidez.

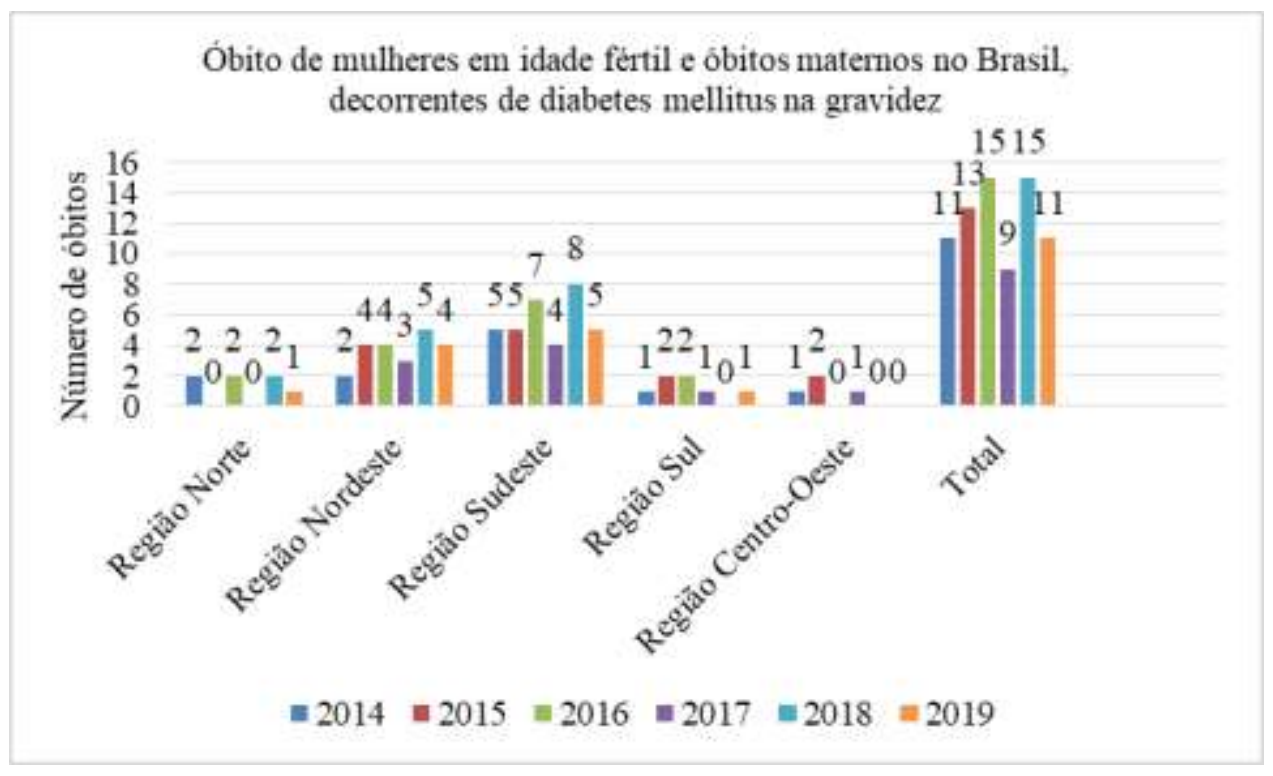

Fonte: MS/SVS/CGIAE - Sistema de Informações sobre Mortalidade - SIM (2021).

\section{Discussão}

A partir das análises utilizadas nesse estudo, verificou-se que, do período de 2014 a 2019, no Brasil, houve uma diminuição da incidência das morbidades decorrentes da gestação. Tal fato pode estar relacionado com a adoção de medidas pelo Ministério da Saúde (MS) que objetivem o acompanhamento do pré-natal, fazendo uso de métodos preventivos, educativos e abordando aspectos psicossociais. Exemplo disso se encontra no Programa de Humanização no Pré-natal e Nascimento (PHPN), proporcionando maior acessibilidade, cobertura e qualidade na assistência à gestante no período prénatal, no parto e pós-parto, e ao recém-nascido (Brasil, 2016).

Sendo assim, o Sul do país apresentou-se com o maior decréscimo no número de morbidades maternas no período estudado, passando de 101.822 em 2014 para 83.347 em 2019, considerando que a estimativa de população geral dessa região aumentou, com 29.016.114 habitantes em 2014, passando para 29.975.984 habitantes em 2019. Esse resultado pode estar relacionado à qualidade de vida mais elevada e aos índices socioeconômicos altos, possibilitando maior acesso aos cuidados pré-natais (Domingues et al., 2015; IBGE, 2019; Jacob et al., 2019).

Além disso, o Sudeste, apesar de apresentar 39,06\% do total dos casos de morbidades no período da gestação, parto e puerpério, é a região mais populosa do Brasil, representando aproximadamente 42,05\% do total, com cerca de 88.371 .433 habitantes em 2019, segundo o Instituto Brasileiro de Geografia e Estatística. Entre 2014 e 2019, houve aumentos populacionais anuais de $0,74 \%, 0,71 \%$ e $0,68 \%, 0,87 \%$ e $0,75 \%$ (IBGE, 2019).

Nas outras regiões (Norte, Nordeste e Centro-Oeste) também houve, inicialmente, diminuição dessas morbidades, notando-se, entretanto, um pequeno aumento dessas prevalências no Norte e no Centro-Oeste entre os períodos de 2016 a 2017. Entre 2017 e 2018, o Nordeste sofreu o maior aumento percentual quanto ao número de casos, totalizando aproximadamente 10,86\%, já o Norte e o Centro-Oeste sofreram acréscimos de 1,54\% e 6,37\%, respectivamente. Entre os anos de 2018 e 2019, as regiões Norte e Nordeste, aumentaram o número de casos em 4,18\% e 3,41\%, porém a região Centro-Oeste sofreu uma pequena diminuição de $0,43 \%$. Em relação às estimativas populacionais durante o estudo, o Nordeste, que abriga cerca de 27,16\% da população do Brasil, com 57.071 .654 habitantes em 2019, apresentou alterações populacionais anuais de $+0,66 \%,+0,63 \%,+0,59 \%,-0,86 \%$ e $+0,55 \%$, entre 2014 e 2019 . Já a população do Norte sofreu aumentos anuais de $1,38 \%$, 
$1,33 \%, 1,28 \%, 1,37 \%$ e $1,37 \%$, e a do Centro Oeste apresentou incrementos de 1,44\%, 1,40\%, 1,36\%, 1,32\% e $1,31 \%$ no período estudado (IBGE, 2019).

Ademais, os dados de mortalidade obtidos a partir do DATASUS mostraram que, entre as causas de óbito relacionadas predominantemente à gravidez, do período de 2014 a 2019, a mais recorrente é a infecção do trato geniturinário (O23). Já o terceiro maior fator que levou ao óbito nesse intervalo de tempo é a diabetes mellitus na gestação. Embora esse distúrbio metabólico não apresente as maiores taxas de mortalidade materna, continua representando riscos à saúde maternofetal, podendo levar ao desenvolvimento de pré-eclâmpsia, doenças cardiovasculares, parto prematuro para as gestantes e nos fetos podem levar ao desenvolvimento de más-formações no sistema esquelético, cardíaco e nervoso e também se elevam as chances de apresentarem macrossomia (Galerneau \& Inzucchi, 2004; Reece, 2010; Mohsin et al., 2016).

No estudo de distribuição regional da mortalidade decorrente de diabetes mellitus na gestação, observou-se uma maior prevalência na região Sudeste, com 34 casos. O Norte e o Sul apresentam o mesmo resultado de 7 óbitos, o Centro-Oeste apresentou o menor resultado com 4 óbitos e o Nordeste 22 no período de 2014 a 2019. No Norte e no Nordeste os resultados não são vistos com grande acurácia, devido ao fato de que a cobertura do SIM/MS não atingir todo esse território (Laurenti et al., 2004).

Por fim, a mortalidade pela diabetes mellitus na gestação (O24) sofreu um aumento gradual de 2014 a 2016 e diminuiu em ordem de $40 \%$ de 2016 a 2017. Já entre 2017 e 2018 aumentou 40\% e no ano de 2019, houve uma diminuição de 26,66\%. Por outro lado, o número de óbitos decorrentes de complicações da gravidez e do parto (O20-O29), segundo o Capítulo XV (Gravidez, parto e puerpério) do CID-10 sofreu uma diminuição no decorrer dos anos estudados.

Dentre as limitações deste estudo, pode-se considerar a indisponibilidade dos dados de mortalidade fornecidos pela plataforma do DATASUS no ano de 2020. Outra limitação está relacionada ao baixo número de óbitos que dificultou, assim, a realização da análise estatística no que se refere à diabetes gestacional.

\section{Considerações Finais}

Após a realização desse trabalho, pode-se concluir que mesmo com a adoção de medidas que visem à melhora da saúde da gestante, como o $5^{\circ}$ Objetivo de Desenvolvimento do Milênio e o Programa de Humanização no Pré-natal e Nascimento, a prevalência das morbimortalidades relacionadas ao período gestacional e ao parto ainda se apresentam em valores elevados, independentemente de ter sofrido reduções.

Nesse estudo, pode-se notar que mesmo a diabetes mellitus na gestação não sendo a maior responsável pelos óbitos no período estudado, é um distúrbio que deve ser tratado e detectado precocemente, devido ao fato de que pode levar ao desenvolvimento de complicações tanto à gestante, quanto ao feto, a curto e longo prazo. Desse modo, torna-se essencial à qualidade de vida e saúde materna e fetal a assistência pré-natal adequada e a sua adesão, diminuindo o risco de complicações gestacionais.

Posteriormente, será possível realizar estudos que analisem com maior foco nas campanhas pré-natal de cada região do Brasil e em sua adesão, para que assim, seja feita uma comparação melhor da influência destas com os índices de morbidade e mortalidade que ocorrem no período de gestação, possibilitando uma análise mais abrangente dos resultados.

\section{Referências}

Battarbee, A. N., Venkatesh, K. K., Aliaga, S., \& Boggess, K. A. (2020). The association of pregestational and gestational diabetes with severe neonatal morbidity and mortality. Journal of Perinatology, 40(2), 232-239.

Brasil. (2012). Ministério da Saúde. Mortalidade Materna no Brasil. Boletim Epidemiológico Secretaria de Vigilância em Saúde. Bol Epidemiol, 43 (1), 1-7. 
Brasil. (2016). Ministério da Saúde. Protocolos da Atenção Básica: Saúde das Mulheres. Ministério da Saúde, 231 p.

Brasil. (2008). Rede Interagencial de Informações para a Saúde. Indicadores e dados básicos para a saúde. Tema do ano: nascimentos no Brasil.

Domingues, R. M. S. M., Viellas, E. F., Dias, M. A. B., Torres, J. A., Theme-Filha, M. M., Gama, S. G. N. D., \& Leal, M. D. C. (2015). Adequação da assistência pré-natal segundo as características maternas no Brasil. Revista panamericana de salud pública, 37, 140-147.

Galerneau, F., \& Inzucchi, SE (2004). Diabetes mellitus na gravidez. Obstetrics and Gynecology Clinics, 31 (4), $907-933$.

Grandi, S. M., Filion, K. B., Yoon, S., Ayele, H. T., Doyle, C. M., Hutcheon, J. A., \& Platt, R. W. (2019). Cardiovascular disease-related morbidity and mortality in women with a history of pregnancy complications: systematic review and meta-analysis. Circulation, 139(8), 1069-1079.

Hunt, D. (2008). American Diabetes Association (ADA) Standards of medical care in diabetes 2008. Diabetes Care, $31,12-54$.

Jacob, L. M. D. S., Mafetoni, R. R., Figueira, M. C., Lopes, M. H. B. D. M., \& Shimo, A. K. K. (2019). Ações educativas para prevenção de complicações relacionadas à gestação. Revista Enfermagem Atual. 87: 25.

Instituto Brasileiro de Geografia e Estatística. (2019). População residente enviada ao Tribunal de Contas da União - 2001-2019. IBGE, 1 p.

Laurenti, R., Jorge, M. H. P. D. M., \& Gotlieb, S. L. D. (2004). A mortalidade materna nas capitais brasileiras: algumas características e estimativa de um fator de ajuste. Revista Brasileira de Epidemiologia, 7, 449-460.

Leite, I. C. G. (2012). Mortalidade materna em cidade-polo de assistência na região Sudeste: tendência temporal e determinantes sociais. Revista Med Minas Gerais, 22(1), 1-128.

Mohsin, F., Khan, S., Baki, M. A., Zabeen, B., \& Azad, K. (2016). Neonatal management of pregnancy complicated by diabetes. J Pak Med Assoc, 66(9), 814.

Moura, B. L. A., Alencar, G. P., Silva, Z. P. D., \& Almeida, M. F. D. (2018). Internações por complicações obstétricas na gestação e desfechos maternos e perinatais, em uma coorte de gestantes no Sistema Único de Saúde no Município de São Paulo, Brasil. Cadernos de Saúde Pública, 34.

Packer, L. W. (2016). Diabetes gestacional. Revista UNIPLAC. Editora Uniplac, 4(1).

Pereira, A. S., Shitsuka, Do. M., Parreira, F. J., \& Shitsuka, R. (2018). Metodologia da Pesquisa Científica-Licenciatura em Computação. Santa Maria.

Reece, E. A. (2010). As consequências fetais e maternas do diabetes mellitus gestacional. The journal of maternal-fetal \& neonatal medicine, 23 (3), $199-203$.

Roma, J. C. (2019). Os objetivos de desenvolvimento do milênio e sua transição para os objetivos de desenvolvimento sustentável. Ciência e cultura, 71(1), 33-39.

Schoenaker, D. A., Mishra, G. D., Callaway, L. K., \& Soedamah-Muthu, S. S. (2016). The role of energy, nutrients, foods, and dietary patterns in the development of gestational diabetes mellitus: a systematic review of observational studies. Diabetes Care, 39(1), 16-23.

Silva, J. M. D. P. D., Fonseca, S. C., Dias, M. A. B., Izzo, A. S., Teixeira, G. P., \& Belfort, P. P. (2018). Conceitos, prevalência e características da morbidade materna grave, near miss, no Brasil: revisão sistemática. Revista Brasileira de Saúde Materno Infantil, 18, 7-35.

Tomasi, E., Fernandes, P. A. A., Fischer, T., Siqueira, F. C. V., Silveira, D. S. D., Thumé, E., \& Facchini, L. A. (2017). Qualidade da atenção pré-natal na rede básica de saúde do Brasil: indicadores e desigualdades sociais. Cadernos de saúde pública, 33(3), 1-11.

Walker, K. C., Arbour, M. W., \& Wika, J. C. (2019). Consolidation of guidelines of postpartum care recommendations to address maternal morbidity and mortality. Nursing for women's health, 23(6), 508-517. 